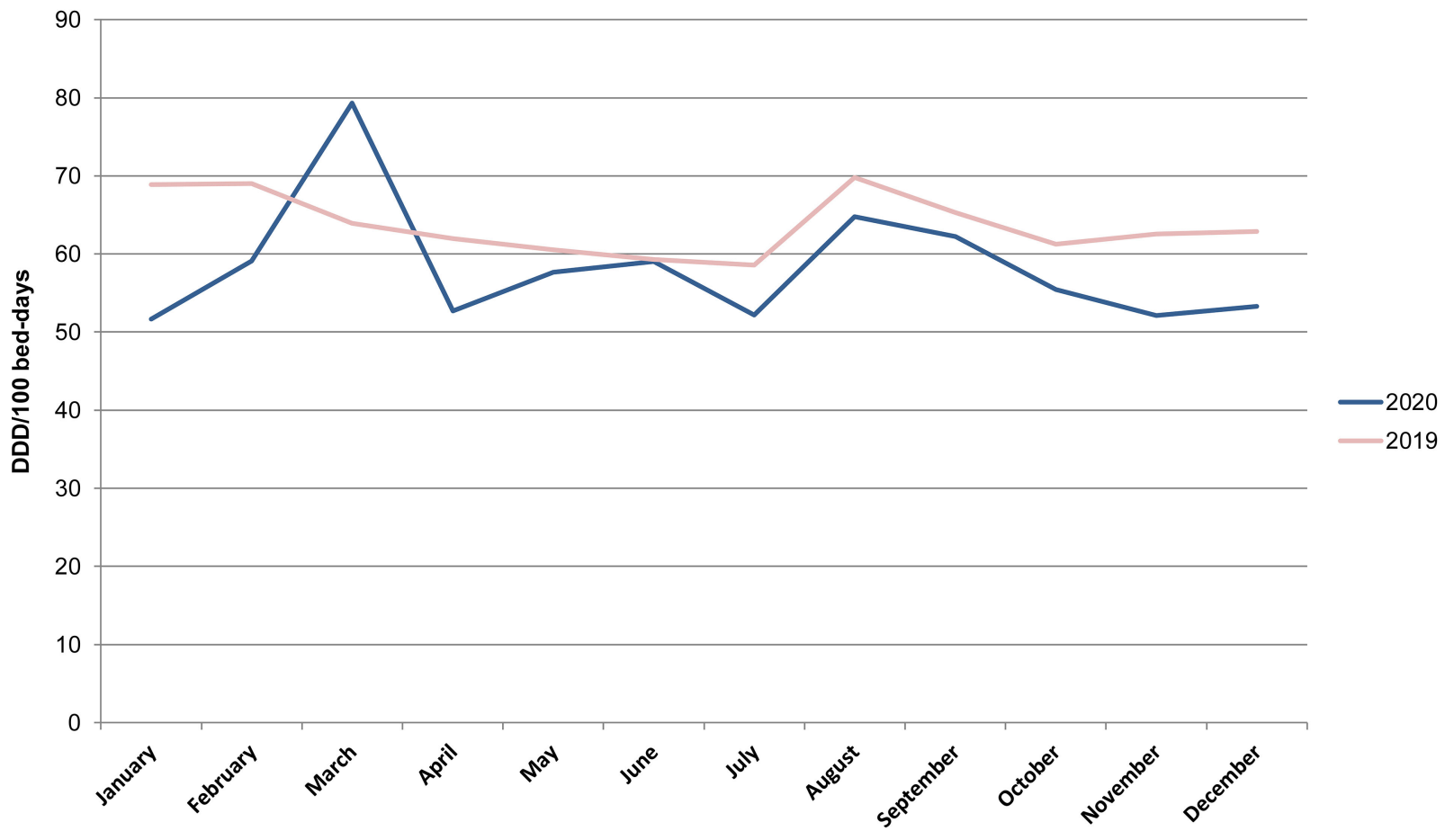

Fig. 1. Global antibiotic consumption.

4. Rawson TM, Moore LSP, Zhu N, et al. Bacterial and fungal coinfection in individuals with coronavirus: a rapid review to support COVID-19 antimicrobial prescribing. Clin Infect Dis 2020;71:2459-2468.

5. Langford BJ, So M, Raybardhan S, et al. Bacterial coinfection and secondary infection in patients with COVID-19: a living rapid review and meta-analysis. Clin Microbiol Infect 2020;26:1622-1629.

6. Rawson TM, Moore LSP, Castro-Sanchez E, et al. COVID-19 and the potential long-term impact on antimicrobial resistance. J Antimicrob Chemother 2020;75:1681-1684.
7. Abelenda-Alonso G, Padullés A, Rombauts A, et al. Antibiotic prescription during the COVID-19 pandemic: a biphasic pattern. Infect Control Hosp Epidemiol 2020;41:1371-1372.

8. Grau S, Echeverria-Esnal D, Gómez-Zorrilla S, et al. Evolution of antimicrobial consumption during the first wave of COVID-19 pandemic. Antibiotics (Basel) 2021;10(2).

\title{
Targeted coronavirus disease 2019 (COVID-19) surveillance for elective procedures: Lessons for resource conservation during a pandemic and beyond
}

\author{
David X. Zheng $\left.\mathrm{BA}^{1}{ }^{(}\right)$, Michelle T. Hecker $\mathrm{MD}^{2}$ and Amy J. Ray MD, $\mathrm{MPH}^{2}$ \\ ${ }^{1}$ Case Western Reserve University School of Medicine, Cleveland, Ohio and ${ }^{2}$ Department of Medicine, The MetroHealth System, Cleveland, Ohio
}

To the Editor-The coronavirus disease 2019 (COVID-19) pandemic has disrupted healthcare delivery worldwide. In mid-March 2020, hospitals across the United States temporarily suspended all elective procedures to mitigate risk of severe acute

Author for correspondence: David X. Zheng, E-mail: dxz281@case.edu

Cite this article: Zheng DX, Hecker MT, and Ray AJ. (2022). Targeted coronavirus disease 2019 (COVID-19) surveillance for elective procedures: Lessons for resource conservation during a pandemic and beyond. Infection Control \& Hospital Epidemiology, 43: 1293-1295, https://doi.org/10.1017/ice.2021.252 respiratory syndrome coronavirus virus 2 (SARS-CoV-2) transmission and to preserve resources to meet clinical demand and ensure workforce preservation (eg, surge planning, employee testing). ${ }^{1,2}$ Although deemed "elective" in that they are nonemergent, elective procedures are essential for patient health and solvency of the nation's healthcare system. ${ }^{3}$

As elective procedures resume, the downstream consequences of their initial delay are becoming apparent. Disruption to patient care may negatively impact outcomes, ${ }^{4}$ and the backlog of elective 


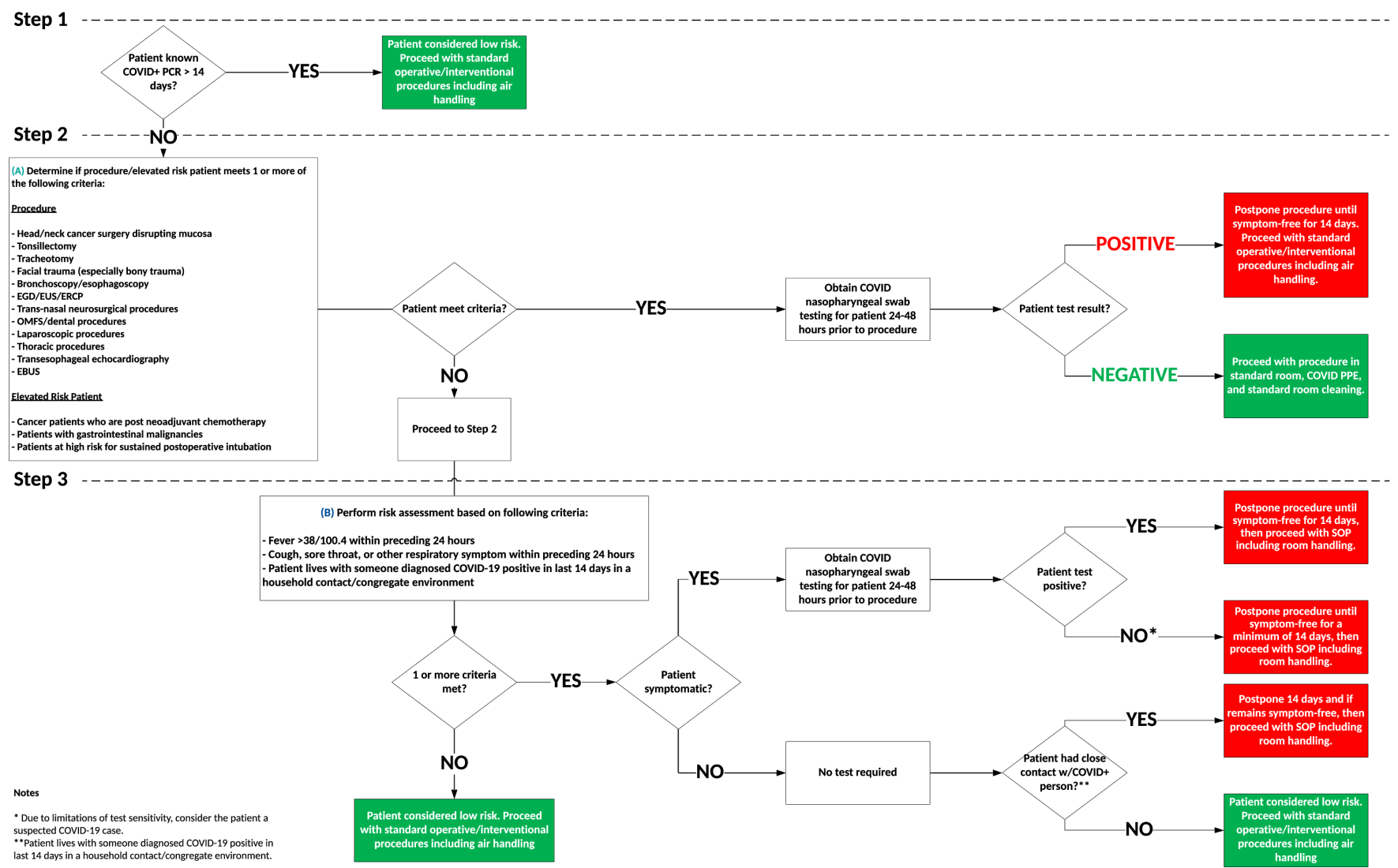

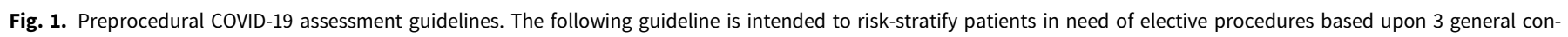

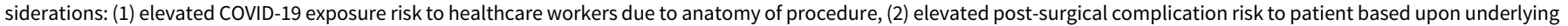
condition, and (3) elevated COVID-19 exposure risk to healthcare workers due to acute respiratory tract infection and/or patient exposure.

procedures needing to be rescheduled will inevitably present a logistical challenge. ${ }^{5}$ Moving forward, it will be important for hospital systems to implement measures balancing infection control with conserving limited resources, meeting patient needs, and preempting additional bottlenecks in scheduling. Given these considerations, we describe our institution's approach to targeted COVID-19 surveillance of patients in need of elective procedures.

The following preprocedural COVID-19 assessment guidelines were implemented at MetroHealth, an urban, safety-net healthcare system located in Cuyahoga County in northeastern Ohio. The first diagnosis of COVID-19 at MetroHealth was made on March 9, 2020, and elective procedures were suspended by the Ohio Department of Health on March 18, 2020. Procedures not requiring hospital stay resumed in late April, and all procedures resumed in early June. ${ }^{6}$

Testing for SARS-CoV-2 was performed with nasopharyngeal swabs in viral transport media. Reverse-transcription polymerase chain reaction (RT-PCR) was performed by the MetroHealth onsite laboratory.

Our preprocedural COVID-19 assessment guidelines are outlined in Figure 1. For patients with a history of COVID-19, assuming completion of isolation ( $>14$ days) prior to intended date of their procedure, persistent contagion or reinfection was deemed unlikely, and no preprocedural testing was pursued.
A risk assessment tool was utilized for patients without prior COVID-19, qualifying risk elevation based upon intended procedure and/or underlying host status. For patients whose procedures involved oral, nasal, esophageal, or thoracic mucosal disruption, we hypothesized that concurrent asymptomatic infection with SARS-CoV-2 could worsen surgical outcome and heighten risk of transmission to the procedural team. ${ }^{7}$ If the preprocedural test was negative, COVID-19 personal protective equipment (PPE) was recommended due to test sensitivity limitations, limited treatment options for acute COVID-19 should transmission to healthcare workers occur, and absence of primary infection prevention in the pandemic's early months. Elevated risk populations included cancer patients undergoing postneoadjuvant chemotherapy, patients with gastrointestinal malignancies, and patients at high risk of sustained postoperative intubation. We hypothesized that asymptomatic infection in this population may complicate postsurgical course due to underlying serious conditions. In addition, for patients in need of solid tumor resection or debulking, we hypothesized that prolonged delay of an intended procedure could worsen cancer outcome. If 1 or more elevated risk criteria were met, a SARS-CoV-2 RT-PCR test was obtained 24-48 hours prior to the procedure.

A symptom assessment tool was utilized for low-risk patients, including the following criteria: fever $>38^{\circ} \mathrm{C}$ within the previous 
24 hours; cough, sore throat, or other respiratory symptoms within the previous 24 hours; and cohabitation with someone diagnosed SARS-CoV-2 positive within the previous 14 days. If none of these criteria were met, the patient was considered low risk and preprocedural testing was not pursued. Symptomatic patients underwent testing for acute COVID-19. Potentially exposed patients were tested only if symptomatic; otherwise, their procedures were delayed to allow adequate incubation time and convalescence.

Targeted COVID-19 surveillance for elective procedures was implemented from May 1 to July 8, 2020. During this time, 3,771 elective procedures were scheduled. Of these cases, 1,049 (27.8\%) qualified for preprocedural SARS-CoV-2 testing according to our surveillance protocol. Among these patients, $19(1.8 \%)$ tested positive.

We assume conservative estimates for sensitivity and specificity of RT-PCR for SARS-CoV-2 at $90 \%$ and $95 \%$, respectively. ${ }^{8}$ Universal screening of 3,771 elective procedures at $3 \%$ prevalence would have resulted in 285 patients delayed at least 2 weeks after suspension of elective procedures. With our protocol, we delayed elective procedures for only 19 patients over 2 months, thus preventing delayed procedural care for 266 patients. Furthermore, given that we used only 1,049 RT-PCR tests, 2,722 were conserved.

Our protocol accounted for potential of elevated infection risk to healthcare workers due to procedural anatomy and patient exposure. Aerosol-generating procedures involving the oral, nasal, esophageal, or thoracic mucosa present elevated risk of SARSCoV-2 exposure to healthcare workers. ${ }^{7}$ Therefore, operative teams donned full PPE with N95 respirator regardless of SARS$\mathrm{CoV}-2$ test result. We also acknowledged that negative test results did not necessarily exclude SARS-CoV-2 infection, given the imperfect sensitivity of RT-PCR testing. ${ }^{8}$

We hypothesized that operating on patients with concurrent COVID-19 mucosal infection could worsen postoperative outcomes. Recent studies have shown high postoperative 30-day mortality rates (15.9\%-23.9\%) in COVID-19 patients undergoing surgery, as well as postoperative pulmonary complications in $51.2 \%$ of patients with perioperative SARS-CoV-2 infection. ${ }^{9,10}$ Additionally, 30-day mortality has been shown to be lower in asymptomatic COVID-19 patients undergoing surgery (5.6\%, compared to $23.1 \%$ in symptomatic patients; $P=.118) .{ }^{10}$ These data support the assumptions upon which our protocol was based.

We employed a strategy allowing for continued operation of elective procedures while mitigating risk for both patients and healthcare workers. We implemented our protocol when regional COVID-19 prevalence was relatively low $(<5 \%),{ }^{6}$ and we acknowledge that adjustments may be necessary with higher SARS-CoV-2 prevalence and improved testing capacity. Our targeted surveillance protocol was successful in that it preserved limited testing capacity while simultaneously preventing a bottleneck in the scheduling of elective procedures.

Acknowledgments. Thank you, Mom and Dad, for your unconditional love and support.

Financial support. No financial support was provided relevant to this article.

Conflicts of interest. All authors report no conflicts of interest relevant to this article.

\section{References}

1. Soltany A, Hamouda M, Ghzawi A, et al. A scoping review of the impact of COVID-19 pandemic on surgical practice. Ann Med Surg (Lond) 2020; 57:24-36.

2. Zheng DX, Jella TK, Mitri EJ, Camargo CA Jr. National analysis of COVID-19 and older emergency physicians. Am J Emerg Med 2020. doi: 10.1016/j.ajem. 2020.10.074.

3. Meredith JW, High KP, Freischlag JA. Preserving elective surgeries in the COVID-19 pandemic and the future. JAMA 2020;324: $1725-1726$.

4. Zheng DX, Cullison CR, Mulligan KM, Scott JF. COVID-19 and the surveillance, epidemiology, and end results program: future considerations for skin cancer research. J Plast Reconstr Aesthet Surg 2021. doi: 10.1016/j.bjps.2021. 03.044.

5. Fu SJ, George EL, Maggio PM, Hawn M, Nazerali R. The consequences of delaying elective surgery: surgical perspective. Ann Surg. 2020;272(2): e79-e80.

6. We have a plan for your procedure. MetroHealth website. https://www. metrohealth.org/simplywell/elective-surgeries-at-metrohealth. Accessed December 21, 2020.

7. Wells M, Jella TK, Zheng DX, et al. Impact of COVID-19 geographic distribution on advanced age plastic surgeons: a cross-sectional analysis. J Plast Reconstr Aesthet Surg 2020. doi: 10.1016/j.bjps.2020.12.049.

8. Boger B, Fachi MM, Vilhena RO, Cobre AF, Tonin FS, Pontarolo R. Systematic review with meta-analysis of the accuracy of diagnostic tests for COVID-19. Am J Infect Control 2021;49:21-29.

9. Collaborative CO. Mortality and pulmonary complications in patients undergoing surgery with perioperative SARS-CoV-2 infection: an international cohort study. Lancet 2020;396:27-38.

10. Carrier FM, Amzallag E, Lecluyse V, et al. Postoperative outcomes in surgical COVID-19 patients: a multicenter cohort study. BMC Anesthesiol 2021;21:15. 